\title{
Overexpression of Forkhead box M1 protein associates with aggressive tumor features and poor prognosis of hepatocellular carcinoma
}

\author{
HONG-CHENG SUN ${ }^{1 *}$, MIN LI $^{3 *}$, JI-LIN LU ${ }^{1}$, DONG-WANG YAN ${ }^{1}$, CHONG-ZHI ZHOU $^{1}$, \\ JUN-WEI FAN ${ }^{1}$, XUE-BIN QIN $^{4}$, HUA-MEI TANG ${ }^{2}$ and ZHI-HAI PENG ${ }^{1}$ \\ Departments of ${ }^{1}$ General Surgery and ${ }^{2}$ Pathology, ${ }^{3}$ Laboratory Center, First People's Hospital, \\ Shanghai Jiao Tong University School of Medicine, Shanghai 200080, P.R. China; \\ ${ }^{4}$ Department of Medicine, Brigham and Women's Hospital, Boston, MA 02115, USA
}

Received January 12, 2011; Accepted February 16, 2011

DOI: $10.3892 /$ or.2011.1230

\begin{abstract}
The aim of this study was to detect the expression of the Forkhead box M1 (FOXM1) protein in human hepatocellular carcinoma (HCC) and to associate FOXM1 expression with clinicopathological features of the patients, and predict the prognosis of patients with FOXM1 expression. Surgical tissue specimens from $151 \mathrm{HCC}$ patients were subjected to a tissue microarray construction and immunohistochemistry analysis of FOXM1 and the proliferation marker proliferating cell nuclear antigen (PCNA). The data showed that the FOXM1 protein was expressed in $59.3 \%$ of the HCC tissues, which was significantly higher compared to that of the surrounding non-tumorous tissues (23.8\%; $\mathrm{P}<0.001)$. Moreover, FOXM1 expression was positively correlated with the labeling index of PCNA $(\mathrm{P}<0.001)$ in $\mathrm{HCC}$ and with aggressive tumor phenotypes, such as larger tumor size, multiple tumors, bilobar involvement, poor tumor cell differentiation, advanced stage and macrovascular invasion $(\mathrm{P}<0.05)$. In addition, HCC patients with FOXM1-positive tumors had a poorer recurrencefree and overall survival after hepatectomy than those with FOXM1-negative tumors. Multivariate Cox regression analysis demonstrated that FOXM1 expression was an independent predictor of unfavorable outcome $(\mathrm{P}<0.05)$. The data from the current study suggest that FOXM1 may play an important role in HCC progression and could be further evaluated as a prognostic biomarker and potential therapeutic target.
\end{abstract}

Correspondence to: Professor Zhi-Hai Peng, Department of General Surgery, or Dr Hua-Mei Tang, Department of Pathology, First People's Hospital, Shanghai Jiao Tong University School of Medicine, Shanghai 200080, P.R. China

E-mail: pengpzh@hotmail.com

E-mail: bellahanson@gmail.com

${ }^{*}$ Contributed equally

Key words: hepatocellular carcinoma, Forkhead box M1, prognosis, tissue microarray

\section{Introduction}

Hepatocellular carcinoma (HCC) is a major health concern with a high morbidity and mortality rate worldwide (1). The incidence of HCC continues to increase due to the prevalence of hepatitis B virus infection in Asian countries and increased hepatitis $\mathrm{C}$ virus infection in the Western world $(1,2)$. For clinical management of HCC, surgical resection is still the mainstay of curative modality to date. Nevertheless, long-term survival of HCC is mainly haunted by postoperative tumor recurrence $(1,3)$. To increase the chances of applying timely treatment for HCC patients, prediction of risk for individual recurrence and subsequent prognosis is of critical importance. It is believed that HCC-associated genes, the intrinsic modulators of cancer biology, may hold considerable promise to accurately assess prognostic predictions of HCC patients and provide for putative therapeutic targets $(4,5)$. With our increasing understanding of molecular events involved in $\mathrm{HCC}$, interest has grown in exploring biological markers for their clinical application.

The evolutionarily conserved forkhead box (Fox) family genes of transcriptional regulators consist of more than 50 mammalian proteins that share homology in the winged helix DNA-binding domain (6-11). Of the Fox members, Forkhead box M1 (FOXM1) protein shows some uniqueness, being abundantly expressed in the transformed cells and a variety of human cancers but undetectable in various differentiated cells and quiescent adult tissues (8-18). It has recently been shown that conditional deletion of FOXM1 expression in mouse hepatocytes resulted in failure in cell proliferation and resistance to liver tumor induction by diethylnitrosamine (DEN)/phenobarbital (PB) (6). Furthermore, either FOXM1 allele deficiency or inhibition of FOXM1 function diminished tumor growth and proliferation in mouse liver tumors (7). In addition, knockdown of FOXM1 by siRNA inhibited proliferation and invasion of human HCC cells in vitro (8). These data strongly suggest that FOXM1 plays a crucial role in hepatocarcinogenesis and tumor progression, which may support the notion that FOXM1 expression could be associated with malignant potential and unfavorable prognosis of HCC. However, to date, there have 
been very few studies that focus on determining FOXM1 expression in human HCC tissue and identification of its potential as a prognostic molecular marker. Therefore, in this study, we analyzed FOXM1 expression and proliferating cell nuclear antigen (PCNA) in a tissue microarray from tissue samples of 151 HCC patients who underwent radical hepatectomy. We then evaluated the associated expression of FOXM1 with labeling index of PCNA and clinicopathological data of the patients and predicted HCC prognosis with FOXM1 expression status.

\section{Materials and methods}

Patients. In this study, we recruited $151 \mathrm{HCC}$ patients who underwent radical hepatectomy at our hospital between May 2001 and November 2008. This study was approved by the Institutional Review Board of Shanghai Jiaotong University. Written consents were obtained from each patient or their guardians for use of the tissue specimens. Formalin-fixed and paraffin-embedded (FFPE) tissue specimens and clinicopathological data from these patients were collected. Clinical diagnosis and tumor differentiation assessment of $\mathrm{HCC}$ were according to the WHO grading criteria (19). Liver function was assessed according to Child-Pugh scoring system (20). Pathological staging was determined according to the AJCC Cancer Staging Manual (21).

Follow-up of patients after surgery. The studied subjects were followed up for 17.8 months (ranged between 1.2-85 months). Tumor relapse was monitored by $\alpha$-fetoprotein test and abdominal ultrasonography every 3 months and by lipiodol computed tomography every 6 months for the first year and once a year thereafter. Cerebral magnetic resonance imaging, positron emission tomography, or radioisotope bone scanner was performed when necessary. Any newborn masses in the involved organ and/ or progressive elevation of $\alpha$-fetoprotein levels after surgery were considered as tumor recurrence. Recurrence-free survival (RFS) was defined as the cumulative time that the patient had survived without tumor relapse from the day of surgery, and recurrence after surgery was considered as the event. Overall survival (OS) was defined as the period from the day of surgery to patient death. Survival until the end of the observation, follow-up interruption or death from a cause other than cancer relapse was considered as a censoring event.

Construction of tissue microarray (TMA). FFPE tissue blocks were retrieved from the archives of our pathology department. Matched pairs of HCC and the surrounding non-tumor FFPE blocks from the aforementioned patients were recruited for TMA construction. Briefly, hematoxylin and eosin stained sections from each block were reviewed and representative regions of the lesion were then selected by two senior pathologists. Two tissue cores each $2.0 \mathrm{~mm}$ in diameter from the patient's FFPE block were punched for a total of eight TMA blocks. Sections of $4 \mu \mathrm{m}$ thickness were prepared and subjected to immnostaining of FOXM1 and PCNA. The procedure of TMA construction was technically assisted by Shanghai Biochip (Shanghai, China).

Immunohistochemistry (IHC). IHC was performed as previously described with minor modifications (23). Briefly, the sections were first deparaffinized in xylene and rehydrated through graded ethanol, then heated in boiling citrate buffer (0.01 M, pH 6.0) for $20 \mathrm{~min}$ in a pressure cooker for antigen retrieval. After endogenous peroxidase activity was blocked with $3 \% \mathrm{H}_{2} \mathrm{O}_{2}$ in PBS, the sections were further incubated with $10 \%$ normal goat serum for $30 \mathrm{~min}$, and followed by incubation with a rabbit polyclonal anti-FOXM1 antibody (sc-502, Santa Cruz Biotechnology, Santa Cruz, CA, USA) at a dilution of 1:50 or a mouse monoclonal anti-PCNA antibody (no. 2586, Cell Signaling Technology, Danvers, MA, USA) diluted at $1: 4000$ at $4^{\circ} \mathrm{C}$ overnight. In the next day, the sections were washed with PBS trice and then incubated with

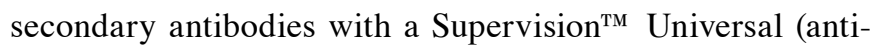
mouse/rabbit) Detection Reagent (HRP) kit (D3004, Shanghai Long Island Biotech, China) according to manufacturer's instructions. Color development was performed using Liquid DAB Substrate Pack (HK520-YAK, Biogenex, San Ramon, CA, USA) and counterstained with Mayer's hematoxylin. Negative control sections were incubated with PBS instead of the primary antibody.

Semi-quantitative assessment of IHC staining. The staining results were independently evaluated by two pathologists (Dr Tang and Dr Jin) without prior knowledge of the patient data. FOXM1 expression was scored as: negative (-), no staining or $<10 \%$ cells with nuclear reactivity and/or weak cytoplasmic staining; positive $(+),>10 \%$ cells with nuclear reactivity and/or distinct cytoplasmic staining. Labeling index of PCNA (PCNA-LI) was defined as the percentage of cells with positive PCNA nuclear staining. PCNA-LI was calculated based on a minimum of 1000 total neoplastic or hepatic cells under high power fields $(\mathrm{x} 400)$ in the representative areas.

When two independent scoring of a case differed, the case was rechecked and a final consensus was achieved by the observers. If the deviation was $<30 \%$ in continuous variable, the mean value was adopted as the final score, otherwise the case was re-evaluated until ultimate agreement was reached.

Statistical analysis. The differences between groups of continuous data were analyzed by t-test and categorical variables were assessed by $\chi^{2}$ or Fisher's exact test. Univariate analysis and survival curves were performed by Kaplan-Meier method and the difference was compared by log-rank test. Cox proportional hazards model was used for multivariate analysis. All the analyses were performed with SPSS 13.0 software. A P-value of $<0.05$ was considered statistically significant.

\section{Results}

Expression of FOXM1 and its association with PCNA-LI. FOXM1 protein on tissue sections was detected by using immunohistochemical staining with a polyclonal anti-FOXM1 antibody. The specific staining for FOXM1 was observed in the cytoplasm and/or nucleus of HCC cells (Fig. 1A and B), but only in cytoplasm of the non-tumorous cells (Fig. 1C). After reviewing all stained TMA sections, one case was excluded due to lack of tumor, and positive FOXM1 expression was present in 89 of 150 cases (59.3\%) (Fig. 1D). In contrast, 36 cases (23.8\%) of the 151 surrounding non-tumorous tissues showed FOXM1 positive (Fig. 1F), indicating that FOXM1 expression 

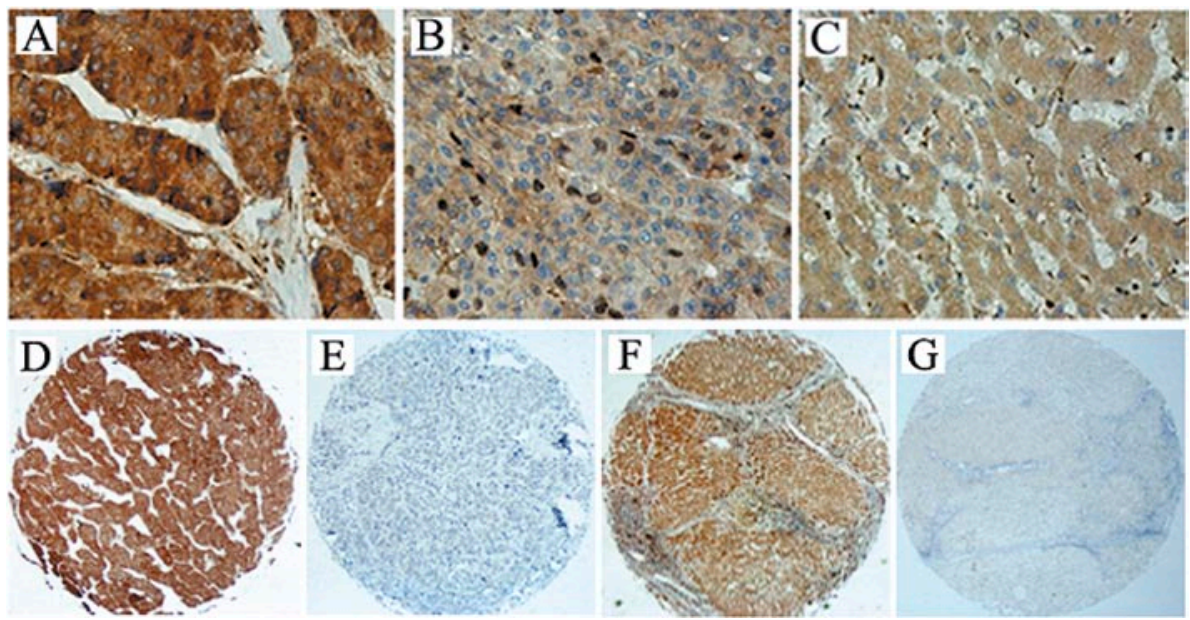

Figure 1. Immunohistochemical analysis of FOXM1 expression in HCC and non-tumorous tissues. (A) FOXM1 immunostaining mainly localized in the cytoplasm of HCC cells (x400). (B) Some of FOXM1 protein can be localized in the nuclei of the malignant cells (x400). (C) Weak cytoplasmic staining of FOXM1 protein in hepatocytes of the non-tumorous tissues (x400). (D) FOXM1 expression in HCC tissue (x40). (E) Lack of FOXM1 staining in HCC tissue (x40). (F) FOXM1 expression in non-tumorous tissue. (G) Lack of FOXM1 staining in the non-tumorous tissue.

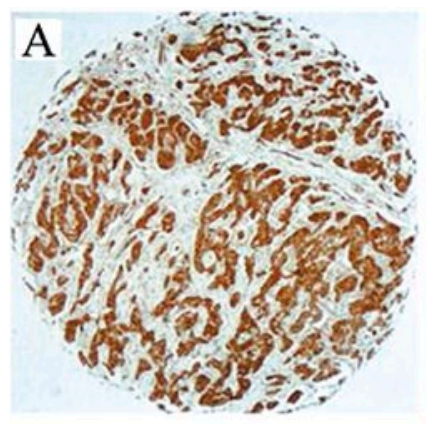

B

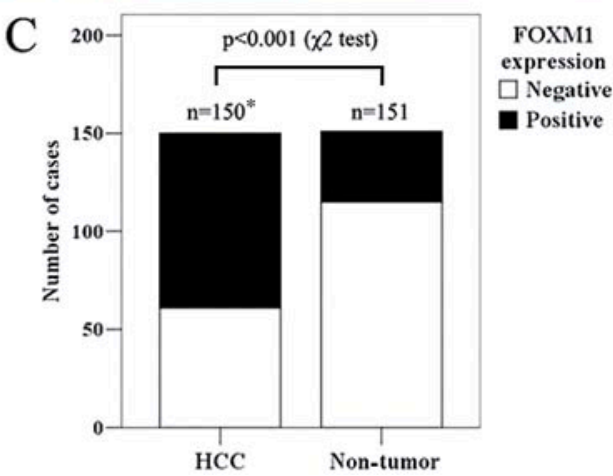

Figure 2. Comparison of FOXM1 expression between HCC and non-cancerous tissues. (A) Positive FOXM1 expression in the tumor (x40). (B) Negative FOXM1 staining in the paired non-tumorous tissue (x40). (C) Comparison of FOXM1 expression between HCC and non-tumorous tissues. ${ }^{*}$ One case of HCC was non-interpretable due to inadequate tumor tissue.

was much higher in tumors than that in the non-tumorous counterparts $(\mathrm{P}<0.001 ;$ Fig. 2). In addition, positive staining of PCNA, a marker of proliferation, was shown mainly in the nuclei of HCC cells (Fig. 3B and D). PCNA labeling index was higher in HCC tissues $(38.25 \pm 27.39 \%)$ than that in the non-tumorous tissues $(27.34 \pm 26.18 \%)$, difference of which is statistically significant $(\mathrm{P}<0.001)$. FOXM1-positive tumors were shown to have higher PCNA labeling index than that of the FOXM1-negative tumors ( $\mathrm{P}<0.001$; Fig. 3).
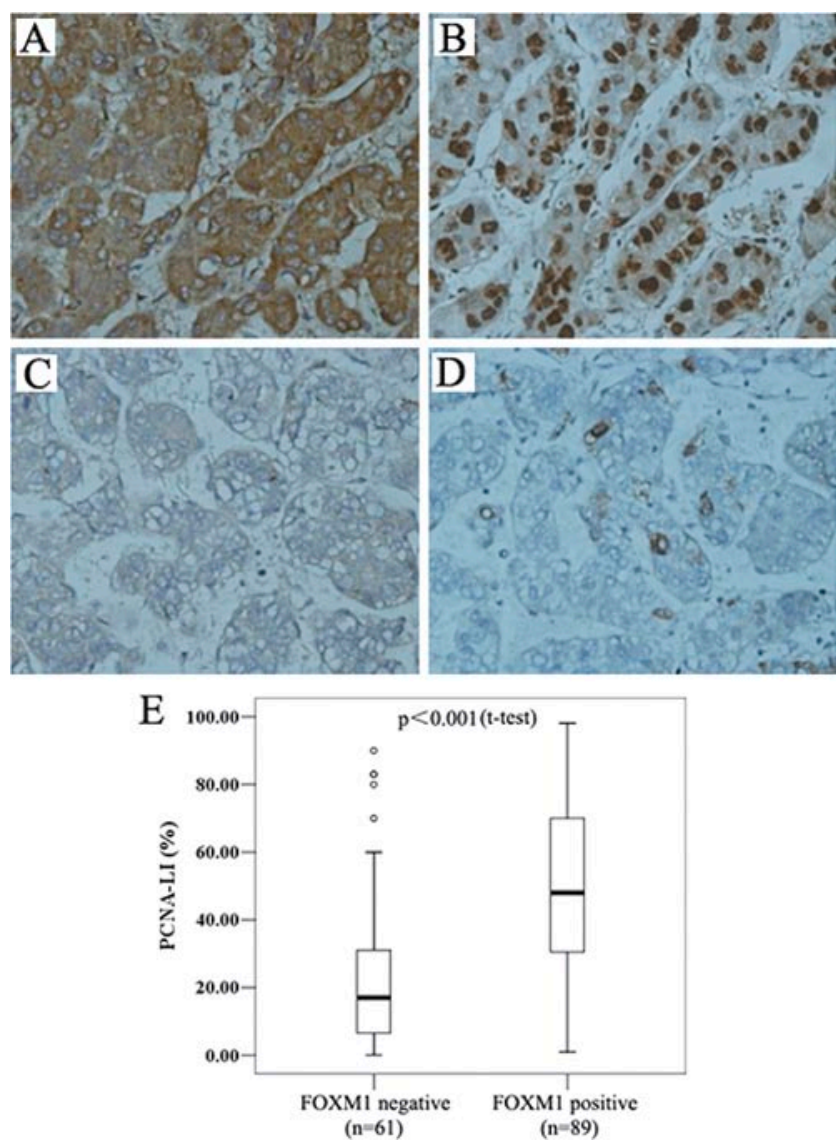

Figure 3. Comparison of PCNA labeling index between FOXM1-positive and negative tumors. FOXM1 positive tumor (A) demonstrated strong nuclear PCNA staining (B) (x400). FOXM1 negative tumor (C) displayed weak nuclear PCNA positivity (D) (x400). (E) FOXM1-positive tumors showed significantly higher PCNA expression than that of FOXM1-negative tumors.

Association of FOXM1 expression with clinicopathological parameters in HCC tissues. As shown in Table I, FOXM1 expression was associated with higher AFP level $(\mathrm{P}=0.034)$, 
Table I. Association between FOXM1 expression and clinicopathological characteristics of HCC patients.

\begin{tabular}{|c|c|c|c|c|c|}
\hline \multirow[b]{2}{*}{ Variables } & \multirow[b]{2}{*}{$\mathrm{N}$} & \multicolumn{2}{|c|}{$\begin{array}{l}\text { FOXM1 } \\
\text { expression }\end{array}$} & \multirow[b]{2}{*}{$\chi^{2}$} & \multirow[b]{2}{*}{ P-value } \\
\hline & & - & + & & \\
\hline Age (years) & & & & 1.354 & $0.245^{\mathrm{a}}$ \\
\hline$\leq 50$ & 75 & 27 & 48 & & \\
\hline$>50$ & 75 & 34 & 41 & & \\
\hline Gender & & & & 0.833 & $0.361^{\mathrm{a}}$ \\
\hline Male & 130 & 51 & 79 & & \\
\hline Female & 20 & 10 & 10 & & \\
\hline $\begin{array}{l}\text { Underlying liver } \\
\text { disease }\end{array}$ & & & & 0.305 & $0.716^{\mathrm{b}}$ \\
\hline $\mathrm{HBV}$ & 142 & 57 & 85 & & \\
\hline Others & 8 & 4 & 4 & & \\
\hline $\begin{array}{l}\text { Liver function } \\
\text { (Child-Pugh) }\end{array}$ & & & & 1.409 & $0.494^{\mathrm{a}}$ \\
\hline Grade A & 75 & 31 & 44 & & \\
\hline Grade B & 60 & 22 & 38 & & \\
\hline Grade C & 15 & 8 & 7 & & \\
\hline $\begin{array}{l}\text { Preoperative AFP } \\
\text { level }\end{array}$ & & & & 4.482 & $0.034^{\mathrm{a}}$ \\
\hline$\leq 200 \mathrm{ng} / \mathrm{ml}$ & 64 & 33 & 31 & & \\
\hline$>200 \mathrm{ng} / \mathrm{ml}$ & 82 & 28 & 54 & & \\
\hline Tumor size & & & & 33.586 & $<0.001^{\mathrm{a}}$ \\
\hline$\leq 8 \mathrm{~cm}$ & 83 & 51 & 32 & & \\
\hline$>8 \mathrm{~cm}$ & 64 & 9 & 55 & & \\
\hline Tumor number & & & & 4.070 & $0.044^{\mathrm{a}}$ \\
\hline$\leq 3$ & 121 & 54 & 67 & & \\
\hline$>3$ & 29 & 7 & 22 & & \\
\hline Tumor distribution & & & & 5.436 & $0.020^{\mathrm{a}}$ \\
\hline Unilobar & 113 & 52 & 61 & & \\
\hline Bilobar & 37 & 9 & 28 & & \\
\hline $\begin{array}{l}\text { Cirrhotic } \\
\text { background }\end{array}$ & & & & 6.414 & $0.011^{\mathrm{a}}$ \\
\hline+ & 137 & 60 & 77 & & \\
\hline- & 13 & 1 & 12 & & \\
\hline $\begin{array}{l}\text { Macrovascular } \\
\text { invasion }\end{array}$ & & & & 12.132 & $<0.001^{\mathrm{a}}$ \\
\hline - & 110 & 54 & 56 & & \\
\hline+ & 40 & 7 & 33 & & \\
\hline Tumor differentiation & & & & 4.138 & $0.042^{\mathrm{a}}$ \\
\hline $\mathrm{G} 1 / \mathrm{G} 2$ & 118 & 53 & 65 & & \\
\hline G3 & 32 & 8 & 24 & & \\
\hline pTNM stage & & & & 19.268 & $<0.001^{\mathrm{a}}$ \\
\hline Stage I/II & 94 & 51 & 43 & & \\
\hline Stage III & 56 & 10 & 46 & & \\
\hline
\end{tabular}

${ }^{\mathrm{a}} \chi^{2}$ test; ${ }^{b}$ Fisher's exact test. larger tumor size $(\mathrm{P}<0.001)$, multiple tumors $(\mathrm{P}=0.044)$, bilobar tumor involvement $(\mathrm{P}=0.020)$, poor tumor differentiation $(\mathrm{P}=0.042)$, non-cirrhotic background $(\mathrm{P}=0.011)$, advanced tumor stage $(\mathrm{P}<0.001)$, and macrovascular invasion $(\mathrm{P}<0.001)$ in HCC tissues.

Association of FOXM1 expression with patient survival. The median time of the follow-up of patient was 17.8 months (ranged between 1.2-85 months). Cumulative 1-, 3- and 5-year recurrence-free survivals (RFS) were 55.5, 43.4 and 38.8\%, respectively, while 1-, 3- and 5-year overall survivals (OS) were $81.4,54.4$ and $48.1 \%$, respectively. Tumor recurrence occurred in $77(51.0 \%)$ patients, among whom 53 (68.8\%) died of the recurrence-related death. Time to the recurrence ranged from 1.0 to 60.3 months after surgery. Six patients who died in less than 1 month due to surgical complications were excluded from survival analysis.

Univariate analysis revealed that the RFS and OS of patients with FOXM1-positive tumors were inferior to those with FOXM1-negative tumors ( $\mathrm{P}<0.001$; Fig. 4 and Table II). Moreover, inferior RFS and OS was observed in patients with younger age $(\mathrm{P}=0.026$ for RFS; $\mathrm{P}=0.005$ for $\mathrm{OS})$, higher pre-operative AFP level ( $\mathrm{P}=0.004$ for RFS; $\mathrm{P}=0.048$ for $\mathrm{OS}$ ), larger tumor size $(\mathrm{P}<0.001$ for both RFS and OS), multiple tumors ( $\mathrm{P}=0.002$ for both RFS and OS), non-cirrhotic background $(\mathrm{P}<0.001$ for RFS; $\mathrm{P}=0.013$ for $\mathrm{OS})$, macrovascular invasion $(\mathrm{P}<0.001$ for both RFS and OS), poor tumor differentiation $(\mathrm{P}<0.001$ for both RFS and OS) or advanced pTNM stage $(\mathrm{P}<0.001$ for both RFS and OS) (Table II).

In addition, we also performed multivariate analysis. All the significant factors in the univariate analysis were included in further multivariate analysis. The data showed that FOXM1 expression $(\mathrm{P}=0.042)$, macrovascular invasion $(\mathrm{P}=0.027)$, tumor differentiation $(\mathrm{P}=0.003)$, pTNM stage $(\mathrm{P}=0.049)$, and cirrhotic background $(\mathrm{P}=0.001)$ maintained independent factors to predict RFS of the patients, whereas FOXM1 expression $(\mathrm{P}=0.003)$, multiple tumors $(\mathrm{P}=0.023)$, macrovascular invasion $(\mathrm{P}<0.001)$, and tumor differentiation $(\mathrm{P}=0.001)$ were independent prognostic factors for the patient OS (Table III).

\section{Discussion}

FOXM1, a typical proliferation-specific transcription factor, plays an important role in cell proliferation and organogenesis in rodents $(9,10)$. Recent studies have shown that FOXM1 was up-regulated in a number of human malignancies and expression of FOXM1 was involved in both tumor initiation and progression $(13,24,25)$. In the context of mouse livers, FOXM1 not only was implicated in diethylnitrosamine/ phenobarbital-induced hepatic carcinogenesis (6), but also required for proliferative expansion during $\mathrm{HCC}$ development (7). Although the pro-oncogenic function of FOXM1 in HCC has been well substantiated by laboratory research, the expression pattern of FOXM1 and its clinical significance is not clearly understood. We found that $59.3 \%$ of the investigated HCC samples showed positive FOXM1 expression compared with $23.8 \%$ of FOXM1-positive non-tumorous tissues, suggesting that overexpression of FOXM1 occurred in human HCC tumors. Moreover, nuclear staining pattern of FOXM1 was observed exclusively in HCC tissues, indicating 

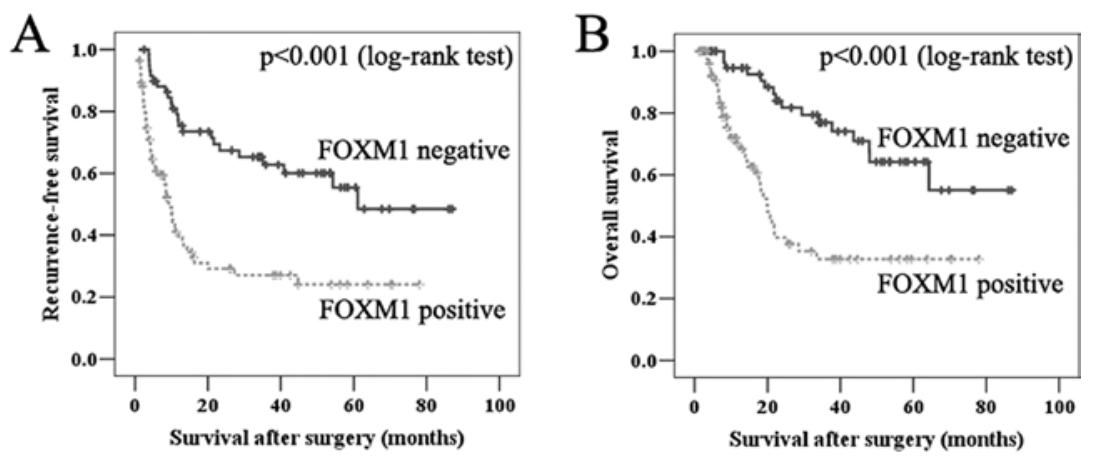

Figure 4. Recurrence-free survival (A) and overall survival (B) curves of HCC patients according to FOXM1 expression.

Table II. Univrariate analysis of variables related to recurrence-free or overall survival of the patients.

\begin{tabular}{|c|c|c|c|c|c|c|c|c|c|c|}
\hline \multirow[b]{2}{*}{ Variables } & \multicolumn{5}{|c|}{ Recurrence-free survival (\%) } & \multicolumn{5}{|c|}{ Overall survival (\%) } \\
\hline & 1 year & 3 years & 5 years & $\chi^{2 \mathrm{a}}$ & $\mathrm{P}$-value ${ }^{\mathrm{a}}$ & 1 year & 3 years & 5 years & $\chi^{2 \mathrm{a}}$ & P-value ${ }^{a}$ \\
\hline Age (years) & & & & 4.931 & 0.026 & & & & 7.990 & 0.005 \\
\hline$\leq 50$ & 43.9 & 37.5 & 33.7 & & & 70.2 & 39.8 & 39.8 & & \\
\hline$>50$ & 65.7 & 49.3 & 44.5 & & & 90.9 & 66.6 & 54.8 & & \\
\hline Preoperative AFP level & & & & 8.117 & 0.004 & & & & 3.895 & 0.048 \\
\hline$>200 \mathrm{ng} / \mathrm{ml}$ & 47.5 & 34.6 & 32.1 & & & 76.3 & 47.1 & 40.4 & & \\
\hline$\leq 200 \mathrm{ng} / \mathrm{ml}$ & 68.8 & 57.6 & 50.9 & & & 87.2 & 65.3 & 58.7 & & \\
\hline Tumor size & & & & 36.101 & $<0.001$ & & & & 29.162 & $<0.001$ \\
\hline$>8 \mathrm{~cm}$ & 30.9 & 20.0 & 12.5 & & & 64.5 & 28.8 & 21.6 & & \\
\hline$\leq 8 \mathrm{~cm}$ & 74.9 & 61.9 & 59.6 & & & 92.9 & 71.8 & 66.3 & & \\
\hline Tumor number & & & & 9.411 & 0.002 & & & & 9.270 & 0.002 \\
\hline$>3$ & 26.2 & 20.9 & 20.9 & & & 70.7 & 19.9 & 19.9 & & \\
\hline$\leq 3$ & 61.9 & 48.5 & 43.0 & & & 85.6 & 63.9 & 57.2 & & \\
\hline Cirrhotic background & & & & 12.266 & $<0.001$ & & & & 6.186 & 0.013 \\
\hline+ & 57.9 & 45.1 & 42.3 & & & 82.7 & 56.3 & 49.6 & & \\
\hline- & 36.9 & 36.9 & 0 & & & 63.0 & 21.0 & 21.0 & & \\
\hline Macrovascular invasion & & & & 64.479 & $<0.001$ & & & & 63.250 & $<0.001$ \\
\hline- & 69.7 & 58.4 & 51.8 & & & 93.5 & 66.5 & 60.5 & & \\
\hline+ & 16.8 & 3.7 & 3.7 & & & 40.3 & 13.8 & 6.9 & & \\
\hline Tumor differentiation & & & & 26.009 & $<0.001$ & & & & 22.966 & $<0.001$ \\
\hline $\mathrm{G} 1 / \mathrm{G} 2$ & 63.7 & 51.3 & 45.5 & & & 89.3 & 60.5 & 55.0 & & \\
\hline G3 & 20.9 & 10.4 & 10.4 & & & 42.2 & 24.6 & 12.3 & & \\
\hline pTNM stage & & & & 54.777 & $<0.001$ & & & & 44.711 & $<0.001$ \\
\hline Stage I/II & 73.8 & 62.1 & 56.8 & & & 96.3 & 69.0 & 62.4 & & \\
\hline Stage III & 25.4 & 11.9 & 7.9 & & & 51.8 & 24.1 & 18.1 & & \\
\hline FOXM1 expression & & & & 20.414 & $<0.001$ & & & & 18.454 & $<0.001$ \\
\hline- & 75.4 & 62.8 & 55.4 & & & 94.5 & 76.9 & 64.2 & & \\
\hline+ & 39.4 & 27.1 & 24.1 & & & 70.2 & 32.8 & 32.8 & & \\
\hline
\end{tabular}

${ }^{\mathrm{a}} \chi^{2}$ and P-value were calculated by log-rank test.

that FOXM1 signaling was more active in tumor cells since nuclear translocation of FOXM1 is required to maximally exert its transcriptional function $(11,26)$. This interpretation is further supported by our finding that FOXM1 expression in HCC was closely correlated with tumor proliferation reflected by nuclear PCNA expression. These ex vivo data suggest that 
Table III. The multivariate analysis for recurrence-free and overall survival of the patients (Backward test).

\begin{tabular}{lccccccrr}
\hline & \multicolumn{3}{c}{ Recurrence-free survival } & & \multicolumn{3}{c}{ Overall survival } \\
\cline { 2 - 3 } Variables & Relative risk & $\chi^{2}$ & P-value & & Relative risk & $\chi^{2}$ & P-value \\
\hline FOXM1 expression (+ vs. -) & 1.769 & 4.122 & 0.042 & & 2.601 & 8.640 & 0.003 \\
Tumor number (>3 vs. $\leq 3)$ & 1.706 & 3.534 & 0.060 & & 2.141 & 5.183 & 0.023 \\
Macrovascular invasion (+ vs. -) & 2.288 & 4.866 & 0.027 & & 4.307 & 22.196 & $<0.001$ \\
Tumor differentiation (G3 vs. G1/G2) & 2.312 & 8.777 & 0.003 & & 2.917 & 10.263 & 0.001 \\
pTNM stage (stage III vs. stage I/II) & 2.233 & 3.875 & 0.049 & & 1.395 & 0.305 & 0.581 \\
Cirrhotic background (- vs. +) & 3.748 & 11.447 & 0.001 & & 2.329 & 3.490 & 0.062 \\
\hline
\end{tabular}

FOXM1 overexpression might represent a potential molecular signature of human HCC.

Previous studies showed that FOXM1 expression was associated with higher histological grade, advanced tumor stage and lymph node metastasis of pulmonary squamous cell carcinoma (16), and late stage of cervical cancer (14). In the present study, we demonstrated a significant correlation between FOXM1 expression and aggressive clinicopathological features of HCC, including higher AFP level, larger tumor size, multiple tumor numbers, bilobar distribution, poor tumor differentiation, macrovascular invasion and advanced stages. This association strongly suggests that FOXM1 could be further evaluated as a biomarker to identify subsets of HCC tumors with aggressive phenotype. Nevertheless, a recent study has documented discrepant data that no significant correlation was observed between FOXM1 expression and clinicopathological parameters in 58 western HCC patients (18). Possible explanations for this discrepancy include differences in detection method for FOXM1 protein, cohort size and patient population between that and our studies.

In our current study of $151 \mathrm{HCC}$ patients, more than half of them developed tumor recurrence that was the predominant cause of death during the follow-up. Therefore, validity of the prognostic value of FOXM1 expression could be at least helpful to identify those patients who would benefit from closer monitoring and from improved treatment strategies. Previous studies demonstrated that FOXM1 expression was inversely associated with survival of HCC patients (18). In line with the current data, we found that expression of FOXM1 was a strong independent predictor of OS for HCC patients. More importantly, our results showed that FOXM1 could also serve as a molecular marker to predict $\mathrm{HCC}$ recurrence and metastasis after liver resection, although further study needs to confirm its usefulness. This finding not only suggests that FOXM1 might help physicians to identify those patients who are at high risk of tumor recurrence, but also provides a foundation for exploring FOXM1-targeted therapy for the future care of $\mathrm{HCC}$ patients.

Another interesting finding in our current study is that HCC patients without cirrhosis had a predominant tendency for tumor recurrence. This propensity may attribute to more aggressive tumor biology manipulated by underlying molecular alterations. The interpretation is supported by the evidence that strong pre-ponderance of these patients with tumors expressing FOXM1 (Table I). However, since there were only 13 patients without cirrhosis, further investigations with more HCC patients will be required to confirm these findings.

$\mathrm{HCC}$ is a disease involving multiple and heterogeneous etiologies that exert differential effects on the molecular events that contribute to HCC development. It is possible that a particular biomarker, which can predict prognosis of HBV-related $\mathrm{HCC}$, may not be relevant for development of HCV-related HCC (4). Considering that our patients were dominated by HBV infection, whether FOXM1 expression can also predict risk of tumor recurrence for patients with HCV-related HCC needs further investigation. For example, further study will clarify whether the altered expression of FOXM1 protein is due to HCC development or HBV infection.

In summary, we demonstrated that FOXM1 was active and aberrantly expressed in human HCC tissues and that FOXM1 overexpression was associated with proliferation and highly aggressive phenotype of HCC. Moreover, FOXM1 expression may be a potential molecular predictor of tumor recurrence and unfavorable clinical outcome in HBV-related HCC patients treated by hepatoectomy. The data from the current study suggest that FOXM1 may play an important role in aggressive progression of human $\mathrm{HCC}$, and that $\mathrm{HCC}$ patients could substantially benefit from FOXM1-targeted therapies. In clinic, a routine evaluation of FOXM1 by immunohistochemistry may improve our insight into the prognosis and treatment of $\mathrm{HCC}$ patients after liver resection.

\section{Acknowledgements}

This study was supported in parts by National Natural Scientific Foundation of China (30700813) and National Key Technology R\&D Program (2008BAI60B03), Ministry of Health, Beijing, China. The authors thank Dr Xiaolong Jin, the head of Department of Pathology, Shanghai Jiao Tong University-Affiliated Ruijin Hospital, for his excellent support and providing technical assistance. We also extend our appreciation to Dr Lei Zhu for the data of patient follow-up and Medjaden Bioscience Limited for assisting in the preparation of this manuscript.

\section{References}

1. Llovet JM, Burroughs A and Bruix J: Hepatocellular carcinoma. Lancet 362: 1907-1917, 2003.

2. EI-Serag HB, Davila JA, Petersen NJ and McGlynn KA: The continuing increase in the incidence of hepatocellular carcinoma in the United States: an update. Ann Intern Med 139: 817-823, 2003. 
3. Chen XP, Qiu FZ, Wu ZD, Zhang ZW, Huang ZY and Chen YF: Long-term outcome of resection of large hepatocellular carcinoma. Br J Surg 93: 600-606, 2006.

4. Mann CD, Neal CP, Garcea G, Manson MM, Dennison AR and Berry DP: Prognostic molecular markers in hepatocellular carcinoma: a systematic review. Eur J Cancer 43: 979-992, 2007.

5. Frau M, Biasi F, Feo F and Pascale RM: Prognostic markers and putative therapeutic targets for hepatocellular carcinoma. Mol Aspects Med 31: 179-193, 2010.

6. Kalinichenko W, Major ML, Wang X, et al: Foxm1b transcription factor is essential for development of hepatocellular carcinoma and is negatively regulated by the p19ARF tumor suppressor. Genes Dev 18: 830-850, 2004.

7. Gusarova GA, Wang IC, Major ML, et al: A cell-penetrating ARF peptide inhibitor of FoxM1 in mouse hepatocellular carcinoma treatment. J Clin Invest 117: 99-111, 2007.

8. Wu QF, Liu C, Tai MH, et al: Knockdown of Fox1M1 siRNA interference decreases cell proliferation, induces cell cycle arrest and inhibits cell invasion in MHCC-97H cells in vitro. Acta Pharmacol Sin 31: 361-366, 2010.

9. Wierstra I and Alves J: FOXM1, a typical proliferation-associated transcription factor. Biol Chem 388: 1257-1274, 2007.

10. Laoukili J, Stahl M and Medema RH: FoxM1: at the crossroads of ageing and cancer. Biochim Biophys Acta 1775: 92-102, 2007.

11. Ye H, Holterman AX, Yoo KW, Franks RR and Costa RH Premature expression of the winged helix transcription factor $\mathrm{HFH}-11 \mathrm{~B}$ in regenerating mouse liver accelerates hepatocyte entry into S phase. Mol Cell Biol 19: 8570-8580, 1999.

12. Teh MT, Wong ST, Neill GW, Ghali LR, Philpott MP and Quinn AG: FOXM1 is a downstream target of Gli1 in basal cell carcinomas. Cancer Res 62: 4773-4480, 2002.

13. Liu M, Dai B, Kang SH, et al: FoxM1B is overexpressed in human glioblastomas and critically regulates the tumorigenicity of glioma cells. Cancer Res 66: 3593-3602, 2006.

14. Chan DW, Yu SY, Chiu PM, et al: Over-expression of FOXM1 transcription factor is associated with cervical cancer progression and pathogenesis. J Pathol 215: 245-252, 2008.

15. Zhang Y, Zhang N, Dai B, et al: FoxM1B transcriptionally regulates vascular endothelial growth factor expression and promotes the angiogenesis and growth of glioma cells. Cancer Res 68: 8733-8742, 2008.

16. Yang DK, Son CH, Lee SK, Choi PJ, Lee KE and Roh MS: Forkhead box M1 expression in pulmonary squamous cell carcinoma: correlation with clinicopatholocial features and its prognostic significance. Human Pathol 40: 464-470, 2009.
17. Zeng $\mathrm{J}$, Wang $\mathrm{L}, \mathrm{Li} \mathrm{Q}$, et al: FoxM1 is up-regulated in gastric cancer and its inhibition leads to cellular senescence, partially dependent on p27kip1. J Pathol 218: 419-427, 2009.

18. Calvisi DF, Pinna F, Ladu S, et al: Forkhead box M1B is a determinant of rat susceptibility to hepatocarcinogenesis and sustains ERK activity in human HCC. Gut 58: 679-687, 2009.

19. Li Q, Zhang N, Jia Z, et al: Critical role and regulation of transcription factor FoxM1 in human gastric cancer angiogenesis and progression. Cancer Res 69: 3501-3509, 2009.

20. Hamilton SR and Aaltonen LA: Pathology and genetics of tumors of the digestive system. In: World Health Organization Classification of Tumors. Kleihues P and Sobin LH (eds). International Agency for Research on Cancer. IARC Press, Lyon, pp159-172, 2000.

21. D'Angelica M and Fong Y: The liver. In: Sabiston Textbook of Surgery: The Biological Basis of Modern Surgical Practice. 18th edition. Townsend CM, Meauchamp RD, Evers BM and Mattox KL (eds). Saunders, An Imprint of Elsevier, Philadelphia, PA, pp1463-1523, 2008.

22. American Joint Committee on Cancer (AJCC): Liver. In: AJCC Cancer Staging Manual 6th Edition. Greene FL, Page DL, Fleming ID, et al (eds). Springer-Verlag New York, Inc., New York, NY, pp131-136, 2002.

23. Sun HC, Qiu ZJ, Liu J, et al: Expression of hypoxia-inducible factor-1 alpha and associated proteins in pancreatic ductal adenocarcinoma and their impact on prognosis. Int J Oncol 30: 1359-1368, 2007.

24. Kalin TV, Wang IC, Ackerson TJ, et al: Increased levels of the FoxM1 transcription factor accelerate development and progression of prostate carcinoma in both TRAMP and LADY transgenic mice. Cancer Res 66: 1712-1720, 2006.

25. Kim IM, Ackerson T, Ramakrishna S, et al: The Forkhead Box $\mathrm{m} 1$ transcription factor stimulates the proliferation of tumor cells during development of lung cancer. Cancer Res 66: 2153-2161, 2006

26. Ma RY, Tong TH, Cheung AM, Tsang AC, Leung WY and Yao KM: Raf/MEK/MAPK signaling stimulates the nuclear translocation and transactivating activity of FOXM1c. J Cell Sci 118: 795-806, 2005. 